\title{
Can One Hear the Dimension of a Fractal?
}

\author{
Jean Brossard ${ }^{1}$ and René Carmona ${ }^{2}$ \\ 1 Institut Fourier, Université de Grenoble 1, B.P. 74, F-38402 Saint Martin d'Heres Cédex, \\ France \\ 2 Department of Mathematics, University of California at Irvine, Irvine, CA92717, USA \\ Dedicated to the memory of Mark Kac
}

\begin{abstract}
We consider the spectrum of the Laplacian in a bounded open domain of $\mathbb{R}^{n}$ with a rough boundary (i.e. with possibly non-integer dimension) and we discuss a conjecture by M. V. Berry generalizing Weyl's conjecture. Then using ideas Mark Kac developed in his famous study of the drum, we give upper and lower bounds for the second term of the expansion of the partition function. The main thesis of the paper is to show that the relevant measure of the roughness of the boundary should be based on Minkowski dimensions and on Minkowski measures rather than on Haussdorff ones.
\end{abstract}

\section{Introduction}

Let $n$ be an integer, let $D$ be a fixed bounded open set in $\mathbb{R}^{n}$, and let us denote by $H_{D}$ ( $H$ when the domain in $D$ is understood) minus one half the Dirichlet Laplacian in $D$. If we consider $H_{D}$ as a self adjoint operator on $L^{2}(D, d x)$, its spectrum is discrete and can be written down in the form

$$
0<\mu_{1}<\mu_{2} \leqq \mu_{3} \ldots \infty \text {. }
$$

We consider the counting function

$$
N_{D}(\mu)=\#\left\{j \geqq 1 ; \mu_{j} \leqq \mu\right\} .
$$

With these notations, Weyl's famous theorem states that:

$$
N_{D}(\mu) \sim c_{n} \mathfrak{R}_{n}(D) \mu^{n / 2},
$$

as $\mu$ tends to $\infty$, where $\mathfrak{Q}_{n}$ denotes the usual Lebesgue measure in $\mathbb{R}^{n}$, and where $c_{n}$ is a universal constant depending only on the dimension $n$. Whenever the boundary of $D$ is smooth, it was even proven that:

$$
N_{D}(\mu)=c_{n} \mathfrak{Q}_{n}(D) \mu^{n / 2}+O\left(\mu^{(n-1) / 2}\right),
$$


see for example [Seeley (1978)], and this last estimate is on the route of the socalled Weyl's conjecture:

$$
N_{D}(\mu)=c_{n} \mathfrak{Q}_{n}(D) \mu^{n / 2}-c_{n}^{\prime} \mathfrak{Q}_{n-1}(\partial D) \mu^{(n-1) / 2}+o\left(\mu^{(n-1) / 2}\right),
$$

which is known to hold in many cases [see for example Kuznetsov (1966), Ivrii (1980) or Melrose (1980)], but also to fail to be true in some other cases as it was shown in Gromes (1966) and Berard (1983). Note that the existing counterexamples are dealing only with curved manifolds while the ones we exhibit in Sect. 2 below are flat since the domains $D$ are subsets of $\mathbb{R}^{2}$.

As explained in Brownell (1957) the counting function $N_{D}(\mu)$ may well have no asymptotic expansion of the above form while some of its integral transforms do. A commonly used one is the Laplace transform

$$
Z(t)=\int e^{-\mu t} d N(\mu) .
$$

It is called the partition function. Weyl's theorem can be rephrased in terms of the partition function as

$$
Z(t) \sim(2 \pi t)^{-n / 2} \mathfrak{I}_{n}(D)
$$

when $t$ tends to 0 . In order to understand our choice of a title for this paper, let us recall Kac's picturesque way to look at the problem and to interpret (1.1) and (1.2): let us think of $D$ as of an $n$-dimensional drum and let us assume that we have perfect ears so that we can hear the fundamental tones of $D$, namely the eigenvalues $\mu_{j}$. Now, can one guess the geometry of the domain, that is the shape of the drum from this knowledge? Formulae (1.1) and (1.2) tell us that one can hear the volume of the domain. Finding the correction terms to (1.2) and identifying the coefficients and the exponents should tell us more about the other geometrical characteristics of $D$.

This new point of view initiated a wave of interest which culminated in the work (McKean and Singer, 1967) in which the interested reader will find other references. As a final remark on this point we will mention that one cannot guess all the characteristics of the drum as shown in Urakawa (1982).

Motivated by problems in physics related to the scattering of waves by fractals, Berry introduced the concept of diffractal and made the following conjecture (of which we will consider only a weaker form since we want our domain $D$ to be a subset of a Euclidean space with an integer number of dimension). Assuming that the boundary of $D$ is no longer smooth and that its Haussdorff dimension is equal to the positive real number $d$, he conjectured in Berry (1979) and Berry (1980) that the following should be true:

$$
N_{D}(\mu)=c_{n} \mathfrak{I}_{n}(D) \mu^{n / 2}-c_{n, d}^{\prime} d-m(\partial D) \mu^{d / 2}+o\left(\mu^{d / 2}\right),
$$

where $c_{n, d}^{\prime}$ is a universal constant depending only on the dimensions $n$ and $d$, and where $d-m$ denotes the $d$-dimensional Haussdorff measure. This conjecture is a generalization of Weyl's one, and as such it cannot be true in full generality. Nevertheless its form is so nice that it cannot but attract inquiring minds. In fact, even though we did not find any evidence in print we know that many people investigated this problem and related ones. For the same reasons as in the smooth case, this problem may be too difficult and one may want to try to investigate the 
asymptotic of the partition function instead. A simple Abelian argument shows that $(1.1)^{\prime}$ implies that:

$$
Z(t)=(2 \pi)^{-n / 2} \mathfrak{Q}_{n}(D) t^{-n / 2}-c_{n, d}^{\prime \prime} d-m(\partial D) t^{-d / 2}+o\left(t^{-d / 2}\right)
$$

for some other universal constant $c_{n, d}^{\prime \prime}$. Section 3 of the present paper is devoted to the investigation of this pre-Tauberian form of the Berry-Weyl conjecture.

As we are about to demonstrate, another good reason to appeal to the partition function is that it is well suited to the use of probabilistic techniques and this idea is also due to Kac. Let us recall for example that the first few terms of the expansion of the partition function have been explicitly computed in the cases of some plane domains in Louchard (1968) by means of this very technique.

The functional calculus on operators tells us that $Z(t)$ is nothing but the trace of the semigroup generated by the operator $H_{D}$. Even for general domains $D$, this semigroup is given by expectations over Brownian paths (see Simon, 1979) in the sense that:

$$
\left[e^{-t H} f\right](x)=\mathbb{E}_{x}\left\{f\left(X_{t}\right) ; t<T_{D}\right\},
$$

where $X_{t}$ denotes the position of the Brownian path at time $t, T_{D}$ the first time the path exits $D$, i.e.

$$
T_{D}=\inf \left\{t>0 ; X_{t} \notin D\right\},
$$

and $\mathbb{E}_{x}$ the expectation over the paths starting from $x$ at time $t=0$ (we will use the notation $\mathbb{P}_{x}$ for the corresponding probability). The above formula is true for many functions $f$ on $D$, in particular those in $L^{2}(D, d x)$, and for all points $x$ in $D$. Consequently the exponential operator (1.4) have the integral kernel:

$$
e^{-t H}(x, y)=(2 \pi t)^{-n / 2} \mathbb{P}_{x}\left\{t<T_{D} \mid X_{t}=y\right\} \exp \left[-|x-y|^{2} / 2 t\right],
$$

which is, for each fixed $t>0$, a jointly continuous function of $x$ and $y$ according to an old result of G. A. Hunt (see Port and Stone, 1978, for example). The partition function can now be written in the convenient form:

$$
Z(t)=(2 \pi t)^{-n / 2} \int_{D} \mathbb{P}_{x}\left\{t<T_{D} \mid X_{t}=x\right\} d x,
$$

and this will be the starting point of our investigations in Sect. 3. To illustrate how convenient this form can be, let's rederive Weyl's theorem. Formula (1.3) above is rewritten:

$$
Z(t)=(2 \pi t)^{-n / 2} \mathfrak{Q}_{n}(D)-(2 \pi t)^{-n / 2} \int_{D} \mathbb{P}_{x}\left\{t>T_{D} \mid X_{t}=x\right\} d x
$$

from which (1.2) easily follows. The latter will give Weyl's theorem via use of a simple Tauberian theorem "à la Karamata." The fact that the integral in (1.4) tends to zero is what Mark Kac calls "not feeling the boundary." Consequently, in order to estimate the second term in this pre-Tauberian form of Weyl's conjecture, we need to control the asymptotic behavior for small time $t$ of the integral

$$
\int_{D} \mathbb{P}_{x}\left\{t>T_{D} \mid X_{t}=x\right\} d x
$$

We end this introduction with a brief discussion of the content of this paper. 
In Sect. 2 we comment on Berry's extension of Weyl's conjecture. We use two examples to try to point out for which domains this conjecture cannot possibly be true. In particular, our examples clearly demonstrate that the Haussdorff dimension and the Haussdorff measure are not appropriate in all the cases we would like to study. In fact, our Example 2 shows that we can have a continuum of counterexamples to the conjecture (1.1)' and of its weaker form (1.2) by exhibiting plane domains for which (1.1)' essentially holds with any real number $d$ between $\log 5 / \log 3$ and 1 while the Haussdorff dimension of $\partial D$ remains equal to $\log 5 / \log 3$ when $d$ varies. It also shows that we have to use another kind of "content" for the boundary of the domain, and this family of counterexamples seems to indicate that a good candidate is the so-called Minkowski one.

This point is carried over in Sect. 3 where we give some positive answers to the questions we raised on the asymptotics of the partition function of fractal domains. We prove a lower bound in the general case and we give an upper bound under some very mild conditions on the roughness of the boundary. The fact that we need some exterior regularity condition on the boundary to control the second term in the asymptotics of the partition function $Z(t)$ should not be surprising. Indeed, we can remove a countable set of points from $D$ without changing either $N(\mu)$ or $Z(t)$, but if these points are judiciously chosen, we can vary the Minkowski dimension and the Minkowski measure at will. We also show that our lower bound and our upper bound are identical in the case of a $C^{1}$ boundary. This yields formula (1.2) with $d=n-1$, and the surface area of the boundary instead of $d-m(\partial D)$. We believe this result is known but we could not find a published proof. Moreover it is interesting to realize it as a by-product of our upper and lower bounds via some elementary estimates on Brownian paths.

Unfortunately our results are not completely satisfactory for the general fractal domains. They merely correct Berry's first attempt which was in a wrong direction as we proved. They also shed some light on the very nature of the problem by formulating and partially proving a conjecture which is more likely to be true. In fact, they should be considered as a first step toward a better understanding of this challenging and difficult problem.

\section{Discussion of the Conjecture}

We present two examples to show that formula (1.1)' does not stand a chance to be true in general. Our examples are very simple. They are designed to lead to a better statement of the conjecture.

Example 1. Let $D^{\sim}$ be the closed disk centered at the origin of $\mathbb{R}^{2}$ and whose measure is 1 , and let $\left\{x_{n} ; n \geqq 1\right\}$ be a dense subset of the interior of $D^{\sim}$. For each $a>0$ we construct by induction a sequence $\left\{O_{n}(a) ; n \geqq 1\right\}$ of open rectangles with arbitrary orientation contained in the interior of $D^{\sim}$, and such that each $O_{n}(a)$ contains $x_{n}$, is of measure smaller than $a 2^{-n}$ and intersects the union of the $O_{m}(a)$ for $m<n$. Then we define the open set $D(a)$ as the union of all the $O_{n}(a)$ for $n \geqq 1$.

$D(a)$ is an open connected set. Its Lebesgue measure is at most $a$. Its boundary is $D^{\Upsilon} \backslash D(a)$, which has Lebesgue's measure at least $1-a$. Consequently, the 
Haussdorff dimension of the boundary is 2 and

$$
2-m(\partial D(a))=\beta_{2} \mathfrak{I}_{2}(\partial D(a)) \geqq \beta_{2}(1-a)
$$

for some positive constant $\beta_{2}$ independent of $a$. This shows that the formula (1.1) cannot be true in the present situation because, by choosing $a$ small enough one can make the second term larger than the first one in absolute value and this would force the number of eigenvalues to be negative.

The moral of this example is that the boundary of the domain cannot be too big, at least not too much bigger than the domain itself.

Example 2. We now present our most important example. It will contain features which will illustrate at the same time why the conjecture $(1.1)^{\prime}$ was thought to be true and why it is actually false. Rather than trying to explain the construction in full detail, we draw part of the domain $D$, simply commenting on the complete construction. But we first pick a non-decreasing sequence $\{\square(n), n \geqq 1\}$ of positive integers.
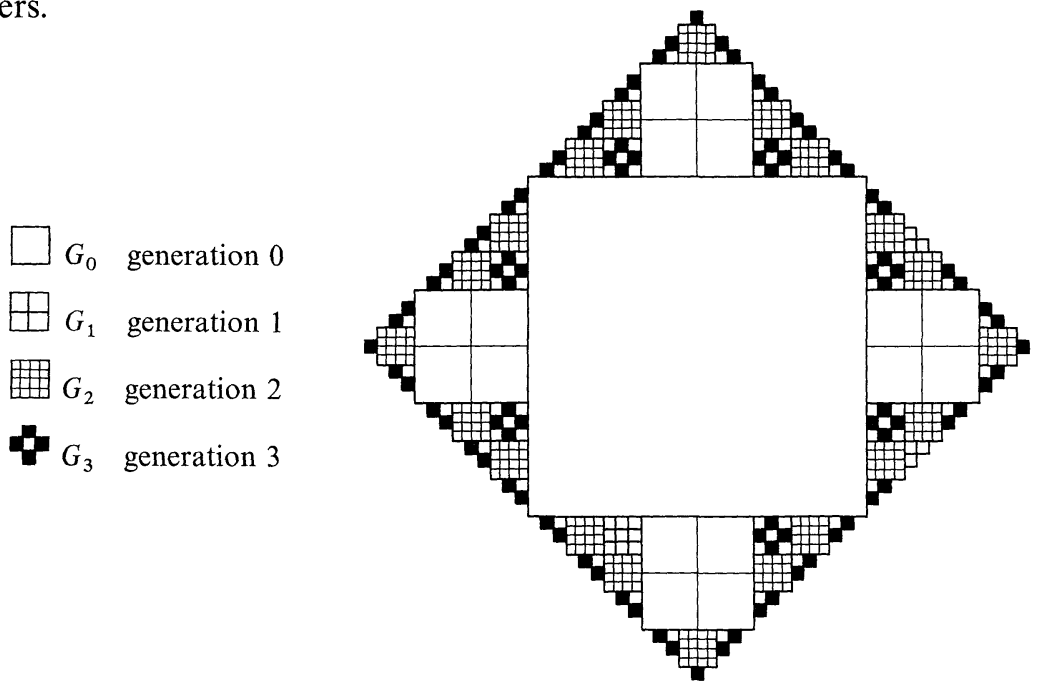

Fig. 1

The generation 0 , say $G_{0}$, contains 1 square of side 1 .

The generation 1 , say $G_{1}$, contains 4 large squares, each of them being of side $1 / 3$ and being divided into $\square(1)^{2}$ identical small squares.

The generation 2 , say $G_{2}$, contains $4 \times 5$ large squares, each of them being of side $3^{-2}$ and being divided into $\square(2)^{2}$ identical small squares.

The generation $n$, say $G_{n}$, contains $4 \times 5^{n-1}$ large squares, each of them being of side $3^{-n}$ and being divided into $\square(n)^{2}$ identical small squares.

The domain $D$ is defined as the union of all the open small squares of all the generations, the square of the $0^{\text {th }}$ generation being considered as small as well as large.

First, we compute the Haussdorff dimension of the boundary $\partial D$.

Let $a>0$ be momentarily fixed (we may think of $a$ as being very small, and in particular smaller than 1) and let $b$ be in $(0, a)$. Let $n=n(a)$ be the integer defined for 
the time being by $[\log (1 / a) / \log 3]$. Recall that we use the notation $[x]$ for the integer part of the nonnegative real number $x . n$ is the smaller integer $k$ satisfying $3^{-k}<a$. We construct a covering of $\partial D$ by open sets with diameters at most $a$ in the following way.

First we put a square of side $3^{-n}$ on each of the 3 active sides of any square of the $n^{\text {th }}$ generation in the following way.

Fig. 2

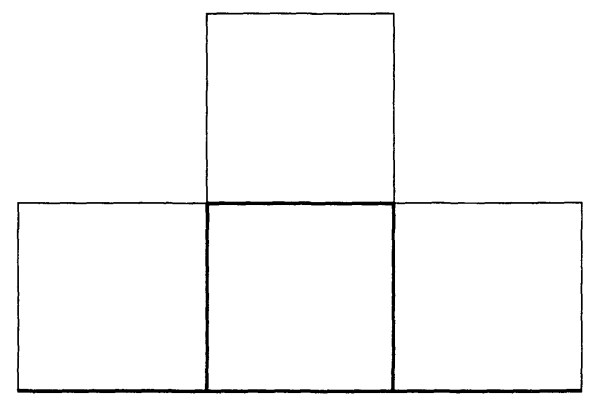

These $3 \times 4 \times 5^{n-1}$ squares cover all the squares of the generations $G_{k}$ with $k>n$.

Second, for each integer $k<n$, we cover the boundaries of all the squares of the $k^{\text {th }}$ generation with open squares of side $b$. There are $4 \times 5^{k-1}$ large squares in the $k^{\text {th }}$ generation, and since each of them is divided into $\square(k)^{2}$ small squares we need at most $2[\square(k)+1] / b 3^{k}$ of such squares of side $b$. Hence, if we call $\left\{U_{j}\right\}_{j}$ the open covering so constructed, and if $d>1$, we have:

$$
\sum\left(\operatorname{diam} U_{j}\right)^{d} \leqq a^{d} 12 \times 5^{n-1}+8 \sum 5^{k-1}[\square(k)+1] 3^{-k} b^{d-1} .
$$

Since we are interested in the infimum $d-m_{\sqrt{2 a}}(\partial D)$ of these quantities over all the coverings by sets of diameters at most $\sqrt{2 a}$ we can take the limit $b \rightarrow 0$ in the righthand side, and this leaves us only with the first term because $d>1$. If we recall that $a$ is of the same order as $1 / 3^{n-1}$ we obtain

$$
d-m_{\sqrt{2 a}}(\partial D) \leqq(36 / 5)\left[5 \times 3^{-d}\right]^{n(a)},
$$

but since $\lim _{a \rightarrow 0} n(a)=+\infty$ this implies:

$$
\lim _{a \rightarrow 0} d-m_{\sqrt{2 a}}\left(\partial D^{\prime}\right) \leqq\left\{\begin{array}{lll}
36 / 5 & \text { if } & d=\log 5 / \log 3 \\
0 & \text { if } & d>\log 5 / \log 3
\end{array}\right.
$$

This shows that the Haussdorff dimension of $\partial D$ is not greater than $\log 5 / \log 3$, and that, its $(\log 5 / \log 3)$-Haussdorff measure is at most $36 / 5$ when we know the Haussdorff dimension is exactly $d=\log 5 / \log 3$, which we prove now by showing that the limit above cannot be zero. By the way, this will also show that the $(\log 5 / \log 3)$-Haussdorff measure of $\partial D$ is not zero. If this limit was 0 , for each $\beta>0$, we could find an $a>0$ and a covering $\left\{F_{1}, \ldots, F_{n}\right\}$ by open sets of diameters $d_{1}, \ldots, d_{n}$ less than $a$ and such that

$$
\sum_{1 \leqq j \leqq n} d_{j}^{d}<\beta
$$

Since we believe no confusion is possible, we use the same notation $n=n(a)$ as before for a different integer. For each $j$ we pick an integer $k(j)$ such that $3^{-[k(j)+1]}$ 
$\leqq d_{j}<3^{-k(j)}$, and we choose an integer $N$ larger than $\max \{k(1), \ldots, k(n)\}$. Let $C_{j}$ be a square of side $3^{-k(j)}$ which contains $F_{j}$. Each large square of the generation $G_{N}$ intersecting $C_{j}$ is contained in the union of at most two large squares of the grid with mesh $3^{-k(j)}$.

Fig. 3

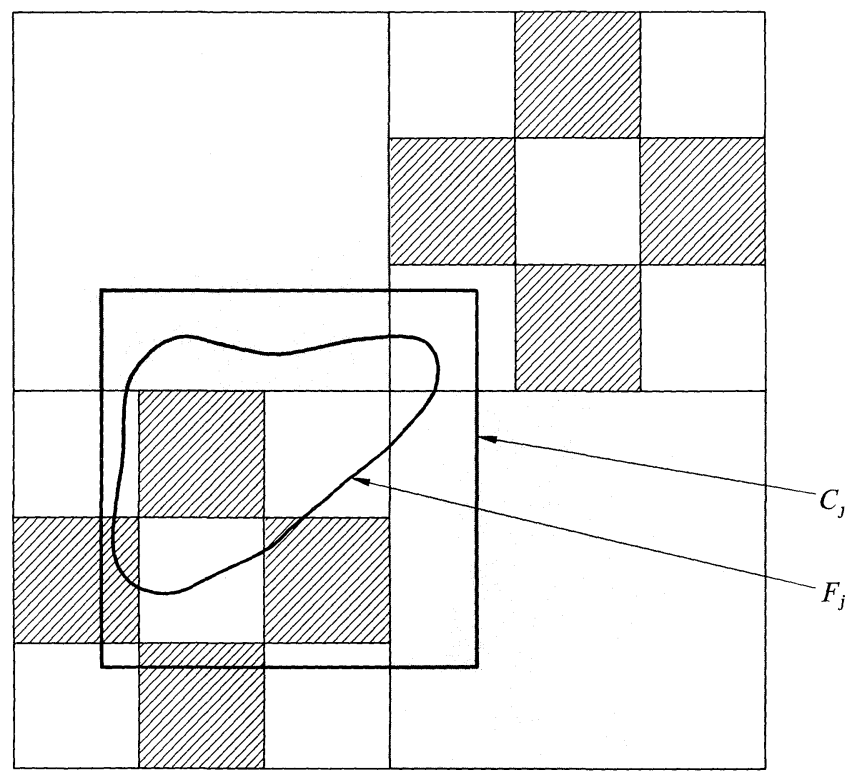

Hence, out of all the large squares of $G_{N}$, at most $2 \times 4 \times 5^{N-k(j)-1}=8 \times 5^{N}$ $\times 3^{-[k(j)+1] d} \leqq 8 \times 5^{N} \times d_{j}^{d}$ of them intersect $C_{j}$, and consequently, $F_{j}$ intersects at most $8 \times 5^{N} \times d_{j}^{d}$ large squares of the generation $G_{N}$ and $\bigcup F_{j}$ intersects at most $8 \times 5^{N} \times \sum d_{j}^{d}<8 \times 5^{N} \times \beta$ large squares of $G_{N}$. This contradicts the fact that $\left\{F_{1}, \ldots, F_{n}\right\}$ is a covering if $\beta$ is small enough. Indeed, if $\beta$ is smaller than $1 / 10$, since $8 / 10<1, \bigcup F_{j}$ cannot even cover the boundary of $G_{N}$.

Notice that this dimension is independent of the sequence $\{\square(n) ; n \geqq 1\}$ which is used in the definition of the domain and on which most of the characteristics of the boundary depend heavily. We now compute the asymptotic of $N(\mu)$; the one of $Z(t)$ will follow with the use of a simple Abelian theorem.

First we recall that if $H=(-1 / 2) d^{2} / d x^{2}$ on some interval $[a, b]$ with Dirichlet boundary conditions at $a$ and $b$, one has:

$$
\Sigma(H)=\left\{k^{2} \pi^{2} / 2(b-a)^{2} ; k=1,2, \ldots\right\},
$$

all the eigenvalues being simple, and if $H_{C}$ is now one half the negative Dirichlet Laplacian on a square $C$ of side $c$ we must have:

$$
\Sigma\left(H_{C}\right)=\left\{\left(h^{2}+k^{2}\right) \pi^{2} / 2 c^{2} ;(h, k) \in \mathbb{N}^{*} \times \mathbb{N}^{*}\right\},
$$

since the variables can be separated. Now, if $C$ is a small square of the $n^{\text {th }}$ generation, its side is $\left(3^{n} \square(n)\right)^{-1}$, and hence the bottom of the spectrum of $H_{C}$ is $\pi^{2}\left(3^{n} \square(n)\right)^{2}$. Consequently, $C$ does not contribute to $N(\mu)$ if $\mu<\pi^{2}\left(3^{n} \square(n)\right)^{2}$. Hence, if we set:

$$
n(\mu)=\sup \left\{n \in \mathbb{N} ; 3^{n} \square(n) \leqq \mu^{1 / 2} / \pi\right\},
$$


we have:

$$
N(\mu)=\sum_{0 \leqq n \leqq n(\mu)} \text { contribution of } G_{n} .
$$

For $n \geqq 1$, the contribution of $G_{n}$ is $4 \times 5^{n-1} \times \square(n)^{2}$ times $N_{C}(\mu)$ for any square $C$ of side $\left(3^{n} \square(n)\right)^{-1}$. For such a square we have according to the Yarnik's formula recalled on p. 722 of [Kuznetsov (1966)]:

$$
\begin{aligned}
N_{C}(\mu)= & \#\left\{(h, k) \in \mathbb{N}^{*} \times \mathbb{N}^{*} ; \pi^{2}\left(3^{n} \square(n)\right)^{2}\left(h^{2}+k^{2}\right) / 2 \leqq \mu\right\} \\
= & \left\{\text { points of } \mathbb{N}^{*} \times \mathbb{N}^{*} \text { in the disk of radius }(2 \mu)^{1 / 2} /\left[\pi 3^{n} \square(n)\right]\right. \\
& \text { centered at the origin }\} \\
= & (1 / 2 \pi)\left(3^{n} \square(n)\right)^{-2} \mu-0_{+}\left[\sqrt{2}(2 / \pi+1 / 2)\left(3^{n} \square(n)\right)^{-1}\right] \mu^{1 / 2},
\end{aligned}
$$

where we use the notation $0_{+}[x]$ to denote a nonnegative number bounded above by $x$. For $n=0$ the contribution of $G_{0}$ is $N_{C}(\mu)$ for any square $C$ of side 1 , and, using again Yarnik's formula we obtain:

$$
\begin{aligned}
N(\mu)= & \mu /(2 \pi)-0_{+}[\sqrt{2}(2+\pi / 2) / \pi] \mu^{1 / 2}+(2 / 5 \pi) \sum_{1 \leqq n \leqq n(\mu)}(5 / 9)^{n} \mu \\
& -0_{+}\left[2(4+\pi) \sqrt{2} / 5 \pi \sum_{1 \leqq n \leqq n(\mu)}(5 / 3)^{n} \square(n)\right] \mu^{1 / 2} .
\end{aligned}
$$

To make our life easier we now choose a particular sequence $\{\square(n) ; n \geqq 1\}$ by setting:

$$
\square(n)=\left[a^{n}\right]
$$

for some fixed real number $a \geqq 1$. This gives:

$$
\begin{aligned}
= & \mu / \pi-0_{+}[\sqrt{2}(2+\pi / 2) / \pi] \mu^{1 / 2}-(1 / 2 \pi)(5 / 9)^{n(\mu)} \mu \\
& -2(4+\pi) \sqrt{2} / 5 \pi 0_{+}\left[5 a /(5 a-3)\left\{(5 a / 3)^{n(\mu)}-1\right\}-(5 / 2) 0_{+}\left[(5 / 3)^{n(\mu)}-1\right]\right] \mu^{1 / 2} .
\end{aligned}
$$

If we use the notation $f(\mu) \asymp g(\mu)$ whenever there exist two finite strictly positive constants $d_{1}$ and $d_{2}$ such that $d_{1} f(\mu) \leqq g(\mu) \leqq d_{2} f(\mu)$ for all $\mu \geqq 0$, we easily see that:

$$
(5 / 9)^{n(\mu)} \asymp \mu^{\log (5 / 9) / 2 \log 3 a} .
$$

Similarly:

$$
(5 a / 3)^{n(\mu)} \asymp \mu^{\log (5 a / 3) / 2 \log 3 a}
$$

and

$$
(5 / 3)^{n(\mu)} \asymp \mu^{\log (5 / 3) / 2 \log 3 a},
$$

which finally gives:

$$
\mu^{d(a) / 2} \asymp \pi^{-1} \mu-N(\mu)
$$

with:

$$
d(a)=\log \left(5 a^{2}\right) / \log 3 a .
$$

Formula (2.1) does not show that $N(\mu)$ has an asymptotic expansion but it gives the exponent of the first correction term in case such an expansion exists. 
Formula (2.2) contradicts Berry's conjecture according to which the exponent should be given by the Haussdorff dimension which we already computed and which coincides with $d(a)$ only in the case $a=1$. To explain this unexpected exponent we now proceed to the introduction of the Minkowski contents (and of the Minkowski dimension) of the boundary $\partial D$.

Let $\partial$ be a fixed nonnegative function on $D$. We should think of $\partial$ as some distance from the point $x$ in $D$ to the boundary $\partial D$, and we are going to use this distance to measure the roughness of the boundary.

For each positive number $u$ we define the set $D_{u}$ as:

$$
D_{u}=\{x \in D ; \partial(x)<u\} .
$$

And for each positive number $d$ we call lower Minkowski content of $\partial D$ the number $(d)-m_{*}(\partial D)$ defined by:

$$
(d)-m_{*}(\partial D)=\liminf _{u \rightarrow 0} u^{-(n-d)} \mathfrak{Q}_{n}\left(D_{u}\right)
$$

and the upper Minkowski content as the number $(d)-m^{*}(\partial D)$ defined by:

$$
(d)-m^{*}(\partial D)=\limsup _{u \rightarrow 0} u^{-(n-d)} \mathfrak{L}_{n}\left(D_{u}\right) \text {. }
$$

$\partial D$ is said to have Minkowski dimension $d$ if:

$$
0<(d)-m_{*}(\partial D) \leqq(d)-m^{*}(\partial D)<\infty .
$$

If this is the case we will say that $\partial D$ is $\boldsymbol{d}$-Minkowski measurable if:

$$
(d)-m_{*}(\partial D)=(d)-m^{*}(\partial D),
$$

the common value being denoted $(d)-m(\partial D)$ and called the $\boldsymbol{d}$-Minkowski measure of $\partial D$.

We now show that the Minkowski dimension of our present domain $D$ is

$$
d(a)=\log \left(5 a^{2}\right) / \log 3 a,
$$

if we choose for function $\partial(x)$ the usual Euclidean distance from $x$ to the boundary. Equation (2.4) is identical to (2.2) and this shed some light on the asymptotic behavior we got for $N(\mu)$ in (2.1). At this point it should be noticed that this dimension $d(a)$, as a function of $a$ varies from $\log 5 / \log 3$ to 2 when $a$ varies from 1 to $+\infty$, while at the same time the Haussdorff dimension remains constant and equal to $\log 5 / \log 3$.

The interesting features of our example are thus twofold: first they prove that Berry's conjecture cannot be true in general (and especially when the Haussdorff and Minkowski dimensions of the boundary do not coincide) and second, they strongly suggest that the roughness of the boundary should be measured with the Minkowski contents rather than with the Haussdorff measures.

To prove (2.4) we first choose $u>0$ and we set:

$$
n(u)=\max \left\{n \in \mathbb{N} ; 2 u<\left(3^{n} \square(n)\right)^{-1}\right\} .
$$

For each small square $C$ of the generation $G_{n}$ we have $\mathfrak{L}_{2}(C)=\mathfrak{L}_{2}\left(C_{u}\right)$ if $n>n(u)$, while

$$
\mathfrak{L}_{2}\left(C_{u}\right)=\left(3^{n}(n)\right)^{-2}-\left(\left(3^{n} \square(n)\right)^{-1}-2 u\right)^{2}
$$


if $1 \leqq n \leqq n(u)$. Consequently,

$$
\begin{aligned}
\mathfrak{I}_{2}\left(D_{u}\right)= & 1-(1-2 u)^{2} \\
& +\sum_{1 \leqq n \leqq n(u)} 4 \times 5^{n-1} \times \square(n)^{2}\left[\left(3^{n} \square(n)\right)^{-2}-\left(\left(3^{n} \square(n)\right)^{-1}-2 u\right)^{2}\right] \\
& +\sum_{n \geqq n(u)+1} 4 \times 5^{n-1} \times \square(n)^{2} \times\left(3^{n} \square(n)\right)^{-2} \\
= & 4 u-4 u^{2}+(16 / 5) \sum_{1 \leqq n \leqq n(u)}(5 / 3)^{n} \square(n) u-(16 / 5) \\
& \cdot \sum_{1 \leqq n \leqq n(u)} 5^{n} \square(n)^{2} u^{2}+(5 / 9)^{n(u)},
\end{aligned}
$$

and, for the same choice $\square(n)=\left[a^{n}\right]$, it is not difficult to show that:

$$
u^{d(a)-2} \mathfrak{Q}_{2}\left(D_{u}\right) \asymp 1,
$$

which proves that the Minkowski dimension of $\partial D$ is $d(a)$. Even though the constants which are implicit in (2.5) are very close, we could not prove that $\partial D$ was actually $d(a)$-Minkowski measurable.

A simple abelian argument gives:

$$
\pi^{-1}-Z(t) \asymp t^{-d(a) / 2},
$$

which is the weaker form of $(1.2)^{\prime}$ we can get from (2.1).

The counter examples we constructed above are not connected open set, and this could be regarded as a weakness of ours. We do not believe so because we can easily modify a little bit the definition of the open set $D$ to make it connected without altering (2.6). Nevertheless we do not know how to control (2.1) in this process.

In any of the large squares of each generation we open segments whose lengths are equal to $10^{6}$ th of the length of the small squares of the same generation in the way shown in the figure below. If $\partial^{\prime}$ denotes the union of $D$ and these little segments, $D^{\prime}$ is an open connected set which contains $D$ and we show that the asymptotic of its partition function is the same as for $D$.

Fig. 4
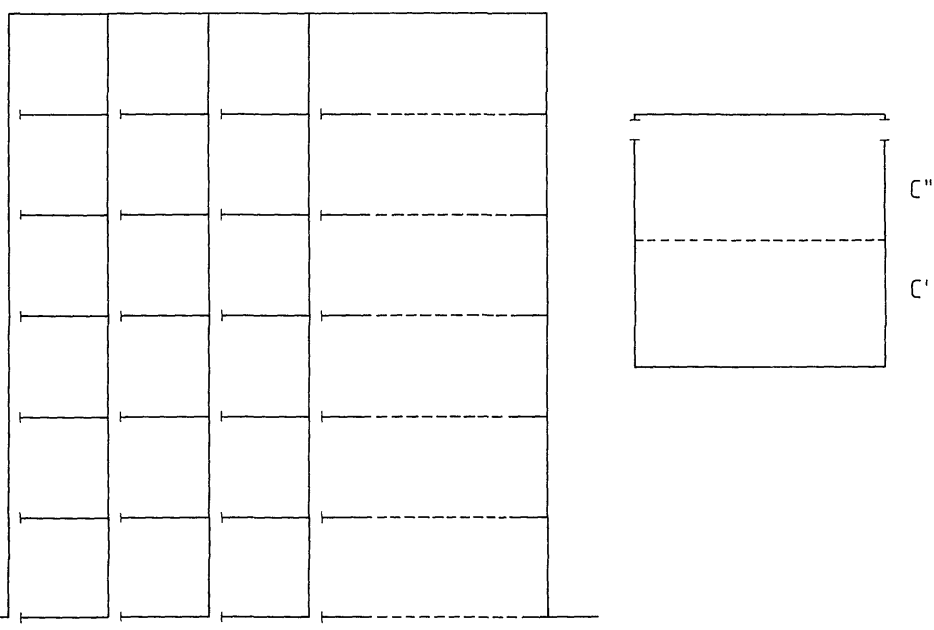
We first note that $T_{D^{\prime}} \geqq T_{D}$ and consequently, for each $x$ in $D$ we have:

$$
\mathbb{P}_{x}\left\{t>T_{D^{\prime}} \mid X_{t}=x\right\} \leqq \mathbb{P}_{x}\left\{t>T_{D} \mid X_{t}=x\right\}
$$

and:

$$
\int_{D^{\prime}} \mathbb{P}_{x}\left\{t>T_{D^{\prime}} \mid X_{t}=x\right\} d x \leqq \int_{D} \mathbb{P}_{x}\left\{t>T_{D} \mid X_{t}=x\right\} d x
$$

On the other hand,

$$
\int_{D} \mathbb{P}_{x}\left\{t>T_{D} \mid X_{t}=x\right\} d x=\sum_{n, C G_{n}} \int_{C} \mathbb{P}_{x}\left\{t>T_{D} \mid X_{t}=x\right\} d x
$$

and:

$$
\int_{C} \mathbb{P}_{x}\left\{t>T_{D} \mid X_{t}=x\right\} d x=\int_{C} \mathbb{P}_{x}\left\{t>T_{C} \mid X_{t}=x\right\} d x=2 \int_{C^{\prime}} \mathbb{P}_{x}\left\{t>T_{C} \mid X_{t}=x\right\} d x
$$

(by symmetry)

$$
\begin{aligned}
= & 2 \int_{C^{\prime}} \mathbb{P}_{x}\left\{t>T_{C}, X\left(T_{C}\right) \in \partial C^{\prime} \mid X_{t}=x\right\} d x \\
& +2 \int_{C^{\prime}} \mathbb{P}_{x}\left\{t>T_{C}, X\left(T_{C}\right) \in \partial C^{\prime \prime} \mid X_{t}=x\right\} d x \\
\leqq & 4 \int_{C^{\prime}} \mathbb{P}_{x}\left\{t>T_{C}, X\left(T_{C}\right) \in \partial C^{\prime} \mid X_{t}=x\right\} d x \\
\leqq & 4 \int_{C^{\prime}} \mathbb{P}_{x}\left\{t>T_{D^{\prime}} \mid X_{t}=x\right\} d x \\
\leqq & 4 \int_{C} \mathbb{P}_{x}\left\{t>T_{D^{\prime}} \mid X_{t}=x\right\} d x,
\end{aligned}
$$

and this shows that

$$
\int_{D^{\prime}} \mathbb{P}_{x}\left\{t>T_{D^{\prime}} \mid X_{t}=x\right\} d x \asymp \int_{D} \mathbb{P}_{x}\left\{t>T_{D} \mid X_{t}=x\right\} d x
$$

\section{Upper and Lower Bounds for the Partition Function}

Let us first decide that the "distance function" $\partial(x)$, with respect to which all the Minkowski contents and dimensions are computed, is from now on, the Euclidean distance from $x$ to the boundary $\partial D$ given by:

$$
\partial(x)=\inf _{z \notin D}\|x-z\| \text {. }
$$

Now, for each $a>0$ we denote by $c_{-}(a)$ the infimum of the positive (and possibly infinite) constants $c$ for which there exists a positive number $u=u(a, c)$ such that:

$$
\mathbb{P}_{x}\left\{t>T_{D} \mid X_{t}=x\right\} \leqq c e^{-a \partial(x)^{2} / t}
$$

for almost every $x$ in $D_{u}$ and all $t>0$. Then, for each $0 \leqq d \leqq n$ we define the constant $c_{-}(n, d)$ by:

$$
c_{-}(n, d)=\inf _{a>0} c_{-}(a) a^{-(n-d) / 2} .
$$


We are now ready to state and prove our lower bound for the partition function $Z(t)$.

Proposition 3.1. With the above notations we have:

$$
\begin{aligned}
Z(t) \geqq & (2 \pi)^{-n / 2} \mathfrak{Q}_{n}(D) t^{-n / 2}-c_{-}(n, d)(2 \pi)^{-n / 2} \Gamma(1+(n-d) / 2) \\
& \cdot d-m^{*}(\partial D) t^{-d / 2}+o\left(t^{-d / 2}\right) .
\end{aligned}
$$

Obviously, this lower bound is empty if either $c_{-}(n, d)$ or the $d$-dimensional upper Minkowski content of the boundary is not finite.

Proof. Without any loss of generality we can assume that $(d)-m^{*}(\partial D)$ is finite. Then, for each positive number $a$, setting $u=u\left(a, c_{-}(a)\right)$, we have:

$$
\begin{aligned}
\int_{D} \mathbb{P}_{x}\left\{t>T_{D} \mid X_{t}=x\right\} d x & \leqq c_{-}(a) \int_{D_{u}} e^{-a \partial(x)^{2} / t} d x+\int_{D \backslash D_{u}} \mathbb{P}_{x}\left\{t>T_{D} \mid X_{t}=x\right\} d x \\
& =c_{-}(a) \int_{0}^{\infty} e^{-\mu} \mathfrak{R}_{n}\left(D_{\sqrt{(\mu t / a)}}\right) d \mu+\int_{D \backslash D_{u}} \mathbb{P}_{x}\left\{t>T_{D} \mid X_{t}=x\right\} d x
\end{aligned}
$$

by a simple integration by parts if one recalls the notation $D_{u}$ defined in (2.3). Now, for $x$ in $D \backslash D_{u}$ we have:

$$
\mathbb{P}_{x}\left\{t>T_{D} \mid X_{t}=x\right\} \leqq \mathbb{P}_{0}\left\{\sup _{0 \leqq s \leqq 1}\left|X_{s}\right|>u t^{-1 / 2} \mid X_{1}=0\right\} \leqq c_{1} e^{-u^{2} / t}
$$

for some positive constant $c_{1}$ independent of $u$ and $t$. This implies:

$$
\lim _{t \rightarrow 0} t^{-(n-d) / 2} \int_{D \backslash D_{u}} \mathbb{P}_{x}\left\{t>T_{D} \mid X_{t}=x\right\} d x=0 .
$$

Hence, using Fatou's lemma we obtain:

$$
\begin{aligned}
& \limsup _{t \rightarrow 0} t^{-(n-d) / 2} \int_{D} \mathbb{P}_{x}\left\{t>T_{D} \mid X_{t}=x\right\} d x \\
& \leqq c_{-}(a) a^{-(n-d) / 2} \limsup _{t \rightarrow 0} \int_{0}^{\infty} e^{-\mu} \mathfrak{I}_{n}\left(D_{\sqrt{(\mu t / a)}}\right)(t \mu / a)^{-(n-d) / 2} \mu^{(n-d) / 2} d \mu \\
& \leqq c_{-}(a) a^{-(n-d) / 2} \Gamma(1+(n-d) / 2)(d)-m^{*}(\partial D) .
\end{aligned}
$$

We could use the "limsup" version of Fatou's lemma because the function $u \rightarrow u^{-(n-d) / 2} \mathfrak{Q}_{n}\left(D_{u}\right)$ is bounded. Indeed, it is bounded near 0 because $d \leqq n$ and it is bounded near $+\infty$ because $(d)-m^{*}(\partial D)$ is finite. Consequently we have:

$$
t^{-n / 2} \int_{D} \mathbb{P}_{x}\left\{t>T_{D} \mid X_{t}=x\right\} d x \leqq c_{-}(n, d) \Gamma(1+(n-d) / 2)(d)-m^{*}(\partial D) t^{-d / 2}+o\left(t^{-d / 2}\right)
$$

by minimizing over $a$, and this is (3.4) if we recall (1.5).

Remark 3.2. It is easy to see that:

$$
c_{-}(n, d) \leqq 2^{-(n-d) / 2} .
$$

Indeed, if $a$ is any positive number and if $B(x)$ denotes the open ball centered at $x$ with radius $\partial(x)$, for each $x$ in $D$ we have:

$$
\mathbb{P}_{x}\left\{t>T_{D} \mid X_{t}=x\right\} \leqq \mathbb{P}_{x}\left\{t>T_{B(x)} \mid X_{t}=x\right\}=\mathbb{P}_{0}\left\{\sup _{0 \leqq s \leqq 1}\left|X_{s}\right|>\partial(x) t^{-1 / 2} \mid X_{1}=0\right\}
$$


by shifting and scaling, and this is easily seen to be bounded above by $c e^{-a \hat{\partial}(x)^{2} / t}$ for some finite constant $c>0$ independent of $x$ and $t$ provided $0<a<2$.

To state and prove the upper bound we proceed similarly. For each positive number $a$ we let $c_{+}(a)$ be the supremum of all the nonnegative numbers $c$ for which:

$$
\mathbb{P}_{x}\left\{t>T_{D} \mid X_{t}=x\right\} \geqq c e^{-a \partial(x)^{2} / t}
$$

for almost every $x$ in $D$ and all $t>0$, and then we set:

$$
c_{+}(n, d)=\sup _{a>0} c_{+}(a) a^{-(n-d) / 2},
$$

and in exactly the same way we prove:

Proposition 3.3. With the above notations we have:

$$
\begin{aligned}
Z(t) \leqq & (2 \pi)^{-n / 2} \mathfrak{L}_{n}(D) t^{-n / 2}-c_{+}(n, d)(2 \pi)^{-n / 2} \Gamma(1+(n-d) / 2) \\
& \cdot(d)-m_{*}(\partial D) t^{-d / 2}+o\left(t^{-d / 2}\right) .
\end{aligned}
$$

As before, this bound has no interest unless both $c_{+}(n, d)$ and the $d$-dimensional lower Minkowski content of the boundary are non-zero.

Proof. For each positive number $a$ we have:

$$
\int_{D} \mathbb{P}_{x}\left\{t>T_{D} \mid X_{t}=x\right\} d x \geqq c_{+}(a) \int_{D} e^{-a \partial(x)^{2} / t} d x=c_{+}(a) \int_{0}^{\infty} e^{-\mu} \mathfrak{Q}_{n}\left(D_{\sqrt{(\mu t / a)}}\right) d \mu
$$

as before. Hence, using Fatou's lemma we obtain:

$$
\begin{aligned}
& \liminf _{t \rightarrow 0} t^{-(n-d) / 2} \int_{D} \mathbb{P}_{x}\left\{t>T_{D} \mid X_{t}=x\right\} d x \\
& \geqq c_{+}(a) a^{-(n-d) / 2} \liminf _{t \rightarrow 0} \int_{0}^{\infty} e^{-\mu} \mathfrak{Q}_{n}\left(D_{\sqrt{(\mu t / a)}}\right)(t \mu / a)^{-(n-d) / 2} \mu^{(n-d) / 2} d \mu \\
& \geqq c_{+}(a) a^{-(n-d) / 2} \Gamma(1+(n-d) / 2)(d)-m_{*}(\partial D) .
\end{aligned}
$$

This shows that:

$$
t^{-n / 2} \int_{D} \mathbb{P}_{x}\left\{t>T_{D} \mid X_{t}=x\right\} d x \geqq c_{+}(n, d) \Gamma(1+(n-d) / 2)(d)-m_{*}(\partial D) t^{-d / 2}+o\left(t^{-d / 2}\right)
$$

by taking the supremum of the right-hand side over $a$.

The main drawback of this upper bound is that we do not know if the constant $c_{-}(n, d)$ is finite in general. We will show later that in the case of a $C^{1}$ boundary, not only this constant is finite, but it actually equals $c_{+}(n, d)$ (see Proposition 3.10 below).

For the time being, we give a sufficient condition under which the upper bound (3.7) holds. It is satisfied by many domains with fractal boundaries and in particular, it is satisfied by our main example in Sect. 2. This sufficient condition requires the boundedness below of the so-called capacitary density of the boundary $\partial D$. This type of assumption seems to be of common use in potential theory. See for example Vituskin (1967) or Oksendal (1972). 
Definition 3.4. We will say that the capacitary density of $\partial D$ is bounded below whenever there exists a constant $c_{0}>0$ such that for each positive number $r<\operatorname{diam} D$ and for each $y$ in $\partial D$ we have:

$$
\operatorname{Cap}(\partial D \cap B(y, r)) \geqq c_{0} \operatorname{Cap}(B(y, r)),
$$

where $\operatorname{Cap}(A)$ denotes the Newtonian capacity of the subset $A$ of $\mathbb{R}^{n}$.

Note that this definition remains the same if the diameter diam $\partial D$ is replaced by any smaller strictly positive number $r_{0}$.

From now one we use the notation $\tau_{A}$ for the first hitting time of the set $A$, i.e. the first exit time $T_{A^{c}}$ of the complement $A^{c}$ of $A$.

Recall that, in dimension $n \geqq 3$, for each Borel set $A$, the function $x \rightarrow \mathbb{P}_{x}\left\{\tau_{A}\right.$ $<\infty$ \} is called the equilibrium potential of $A$ because it is the Green potential of a nonnegative measure called the equilibrium measure of $A$ and denoted $\mu_{A}$, i.e.

$$
\mathbb{P}_{x}\left\{\tau_{A}<\infty\right\}=\int g(x, y) \mu_{A}(d y)
$$

with:

$$
g(x, y)=C_{n}\|x-y\|^{-(n-2)}
$$

for some constant $C_{n}$ depending only on $n . \mu_{A}$ is concentrated on $A$ and the Newtonian capacity of $A$ is nothing but the total mass of this equilibrium measure.

Note that, in dimension $n \geqq 3$, the right-hand side of (3.8) is equal to $c_{0}^{\prime} r^{n-2}$ for some positive constant $c_{0}^{\prime}$ independent of $y$ and $r$. Moreover, the boundedness below of the capacitary density of $\partial D$ implies the existence of a positive constant $c_{0}^{\prime \prime}$ independent of $x$ in $D$ such that:

$$
\mathbb{P}_{x}\left\{\tau_{\partial D \cap B(x, 2 \partial(x))}<\infty\right\} \geqq c_{0}^{\prime \prime} .
$$

Indeed, under this assumption we have:

$$
\begin{aligned}
\mathbb{P}_{x}\left\{\tau_{\partial D \cap B(x, 2 \partial(x))}<\infty\right\} & =C_{n} \int\|x-y\|^{-(n-2)} \mu_{\partial D \cap B(x, 2 \partial(x))}(d y) \\
& \geqq C_{n} \partial(x)^{-(n-2)} \operatorname{Cap}(\partial D \cap B(x, 2 \partial(x))) \\
& \geqq C_{n} \partial(x)^{-(n-2)} \operatorname{Cap}(\partial D \cap B(y, \partial(x)))
\end{aligned}
$$

for any $y$ in $\partial D$ for which $\|x-y\|=\partial(x)$,

$$
\geqq C_{n} c_{0}^{\prime}
$$

because of (3.8) and the above remark.

In dimension $n=2$, the equilibrium measure is the unique probability measure concentrated on $A$ for which the function

$$
u_{A}(x)=\int g(x, y) \mu_{A}(d y)
$$

with

$$
g(x, y)=-\pi^{-1} \log \|x-y\|
$$

is constant on the set $A^{r}$ of regular points for $A$. The function $u_{A}$ is called the equilibrium potential of $A$ and its value on $A^{r}$, say $R(A)$, is called the Robin constant of $A$. The Newtonian capacity of $A$ (also called the Logarithmic capacity 
of $A$ ) is then defined as

$$
\operatorname{Cap}(A)=e^{-R(A)}
$$

For more information the reader is referred to Port and Stone (1978) for example.

Our key estimate is contained in the following:

Lemma 3.5. Let us assume that the capacitary density of $\partial D$ is bounded below. Then, there exist positive constants $c_{1}$ and $C$ such that we have:

$$
\mathbb{P}_{x}\left\{\tau_{\partial D \cap B(x, 2 \partial(x))}<t / 2\right\} \geqq c_{1}
$$

whenever $t>0$ and $x \in D$ are such that $C \partial(x) \leqq \sqrt{t}$.

Proof. Let us assume momentarily the existence of a positive constant $c_{2}$ such that:

$$
\mathbb{P}_{x}\left\{\tau_{\partial D \cap B(x, 2 \partial(x))}<T_{B(x, 6 \partial(x))}\right\} \geqq c_{2}
$$

for all $x$ in $D$. Then, we set $c_{1}=c_{2} / 2$ and we choose $C>0$ such that

$$
\mathbb{P}_{x}\left\{T_{B(x, 6 \partial(x))} \geqq t / 2\right\} \leqq c_{1}
$$

for all $t>0$ and $x$ in $D$ such that $C \partial(x) \leqq \sqrt{t}$. Note that a simple scaling argument shows that $C$ is independent of $t$ and $x$, and depends only on $c_{1}$. Since we obviously have:

$$
\mathbb{P}_{x}\left\{\tau_{\partial D \cap B(x, 2 \partial(x))}<T_{B(x, 6 \partial(x))}\right\} \leqq \mathbb{P}_{x}\left\{\tau_{\partial D \cap B(x, 2 \partial(x))}<t / 2\right\}+\mathbb{P}_{x}\left\{T_{B(x, 6 \hat{(x)})} \geqq t / 2\right\},
$$

we must also have (3.12) if one recalls (3.13) and (3.14) and the definition of $c_{1}$.

Consequently, the proof of the lemma reduces to the proof of (3.13). The latter requires different arguments when $n \geqq 3$ and when $n=2$.

We first assume $n \geqq 3$. Using the strong Markov property one gets:

$$
\begin{aligned}
\mathbb{P}_{x}\left\{\tau_{\partial D \cap B(x, 2 \partial(x))}<\infty\right\}= & \mathbb{P}_{x}\left\{\tau_{\partial D \cap B(x, 2 \partial(x))} \leqq T_{B(x, 6 \partial(x))}\right\} \\
& +\mathbb{P}_{x}\left\{\tau_{\partial D \cap B(x, 2 \partial(x))}<\infty, \tau_{\partial D \cap B(x, 2 \partial(x))} \geqq T_{B(x, 6 \partial(x))}\right\} \\
= & \mathbb{P}_{x}\left\{\tau_{\partial D \cap B(x, 2 \partial(x))} \leqq T_{B(x, 6 \partial(x))}\right\} \\
& +\mathbb{E}_{x}\left\{\mathbb{P}_{X\left(T_{B(x, 60(x))}\right.}\left\{\tau_{\partial D \cap B(x, 2 \partial(x))}<\infty\right\}\right\} \\
\leqq & \mathbb{P}_{x}\left\{\tau_{\partial D \cap B(x, 2 \partial(x))} \leqq T_{B(x, 6 \partial(x))}\right\} \\
& +(1 / 2) \mathbb{P}_{x}\left\{\tau_{\partial D \cap B(x, 2 \partial(x))}<\infty\right\}
\end{aligned}
$$

if we notice that:

$$
\begin{aligned}
\mathbb{P}_{y}\left\{\tau_{\partial D \cap B(x, 2 \partial(x))}<\infty\right\} & =C_{n} \int\|y-z\|^{-(n-2)} \mu_{\partial D \cap B(x, 2 \hat{\partial}(x))}(d z) \\
& \leqq(1 / 2) C_{n} \int\|x-z\|^{-(n-2)} \mu_{\partial D \cap B(x, 2 \partial(x))}(d z)
\end{aligned}
$$

because $\|y-z\| \geqq 2\|x-z\|$ whenever $\|y-x\|=6 \partial(x)$ and $z$ belongs to $\partial D \cap B(x, 2 \partial(x))$,

$$
=(1 / 2) \mathbb{P}_{x}\left\{\tau_{\partial D \cap B(x, 2 \partial(x))}<\infty\right\} .
$$

Finally, (3.15) and (3.11) give (3.13). 
In the case $n=2$, we note that our assumption implies:

$$
R(\partial D \cap B(x, 2 \partial(x))) \leqq \pi^{-1}\left[\log (1 / 2 r)+\log \left(1 / c_{0}\right)\right] .
$$

Fix $m=\pi^{-1} \log (1 / 3 r)$. Notice that:

and that:

$$
\begin{aligned}
\sup _{x \in \partial B(x, 6 \partial(x))} u_{\partial D \cap B(x, 2 \partial(x))}(x) & \leqq \pi^{-1} \log (1 / 4 r) \int_{\partial D \cap B(x, 2 \partial(x))} \mu_{\partial D \cap B(x, 2 \partial(x))}(d y) \\
& =\pi^{-1} \log (1 / 4 r),
\end{aligned}
$$

$$
R(\partial D \cap B(x, 2 \partial(x))) \geqq R\left(B(x, 2 \partial(x))=\pi^{-1} \log (1 / 2 r),\right.
$$

so that:

$$
\sup _{y \in \partial B(x, 6 \partial(x))} u_{\partial D \cap B(x, 2 \partial(x))}(y)<m<R(\partial D \cap B(x, 2 \partial(x))) .
$$

The function

$$
y \rightarrow[R(\partial D \cap B(x, 2 \partial(x)))-m]^{-1}\left[u_{\partial D \cap B(x, 2 \partial(x))}(y)-m\right]
$$

is superharmonic (and hence finely continuous), harmonic on $B(x, 6 \partial(x)) \backslash\{\partial \operatorname{Dn} B(x, 2 \partial(x))\}$, equal to 1 on $A^{r}$ and negative on $\partial B(x, 6 \partial(x))$. Consequently, for $y$ in $B(x, 6 \partial(x)) \backslash\{\partial D n B(x, 2 \partial(x))\}$ we must have:

$$
\begin{aligned}
& {[R(\partial D \cap B(x, 2 \partial(x)))-m]^{-1}\left[u_{\partial D \cap B(x, 2 \partial(x))}(y)-m\right] } \\
&=[R(\partial D \cap B(x, 2 \partial(x)))-m]^{-1} \\
& \quad \cdot \mathbb{E}_{y}\left\{\left[u_{\partial D \cap B(x, 2 \partial(x))}\left(X\left(\min \left(T_{\partial D \cap B(x, 2 \partial(x))}, T_{B(x, 6 \partial(x))}\right)\right)\right)-m\right]\right\} \\
& \leqq \mathbb{P}_{y}\left\{T_{\partial D \cap B(x, 2 \partial(x))}<T_{B(x, 6 \partial(x))}\right\}
\end{aligned}
$$

and consequently,

$$
\begin{aligned}
\mathbb{P}_{x}\left\{\tau_{\partial D \cap B(x, 2 \partial(x))}<T_{B(x, 6 \partial(x))}\right\} & \geqq[R(\partial D \cap B(x, 2 \partial(x)))-m]^{-1}\left[u_{\partial D \cap B(x, 2 \partial(x))}(x)-m\right] \\
& \geqq\left[\log (3 / 2)+\log \left(1 / c_{0}\right)\right]^{-1} \log (3 / 2)
\end{aligned}
$$

if one uses (3.16), the fact that $u_{\partial D \cap B(x, 2 \partial(x))}(x) \geqq \pi^{-1} \log (1 / 2 r)$, and if one recalls that $m=\pi^{-1} \log (1 / 3 r)$. The proof is now complete.

The previous lemma was intended to give the following:

Lemma 3.6. Let us assume that the capacitary density of $\partial D$ is bounded below. Then, there exist positive constants $c_{2}$ and $C$ such that we have:

$$
\mathbb{P}_{x}\left\{T_{D}<t \mid X_{t}=x\right\} \geqq c_{2}
$$

whenever $t>0$ and $x$ in $D$ are such that $C \partial(x) \leqq \sqrt{t}$.

Proof. For each $R>0$ we have:

$$
\begin{aligned}
\mathbb{P}_{x}\left\{T_{D}<t \mid X_{t}=x\right\} & \geqq \mathbb{P}_{x}\left\{\tau_{\partial D \cap B(x, 2 \partial(x))}<t / 2 \mid X_{t}=x\right\} \\
& \geqq \mathbb{P}_{x}\left\{\tau_{\partial D \cap B(x, 2 \partial(x))}<t / 2,\left|x-X_{t / 2}\right| \leqq R \mid X_{t}=x\right\} \\
& =2^{n / 2} \mathbb{E}_{x}\left\{\exp \left[-\left|x-X_{t / 2}\right|^{2} / t\right] ; \tau_{\partial D \cap B(x, 2 \partial(x))}<t / 2,\left|x-X_{t / 2}\right| \leqq R\right\} \\
& \geqq 2^{n / 2} \exp \left[-R^{2} / t\right] \mathbb{P}_{x}\left\{\tau_{\partial D \cap B(x, 2 \partial(x))}<t / 2,\left|x-X_{t / 2}\right| \leqq R\right\},
\end{aligned}
$$


and choosing $R=\alpha \sqrt{t}$ with $\alpha$ such that

$$
\mathbb{P}_{x}\left\{\left|x-X_{t / 2}\right| \geqq \alpha \sqrt{t}\right\}=\mathbb{P}_{0}\left\{\left|X_{t / 2}\right| \geqq \alpha\right\} \leqq c_{1} / 2,
$$

where $c_{1}$ is the constant of Lemma 3.5, we obtain:

$$
\begin{aligned}
\mathbb{P}_{x}\left\{T_{D}<t \mid X_{t}=x\right\} & \geqq 2^{n / 2} \exp \left[-\alpha^{2}\right] \mathbb{P}_{x}\left\{\tau_{\partial D \cap B(x, 2 \partial(x))}<t / 2,\left|x-X_{t / 2}\right| \leqq R\right\} \\
& \geqq 2^{n / 2} \exp \left[-\alpha^{2}\right]\left(\mathbb{P}_{x}\left\{\tau_{\partial D \cap B(x, 2 \partial(x))}<t / 2\right\}-\mathbb{P}_{x}\left\{\left|x-X_{t / 2}\right| \leqq R\right\}\right) \\
& \geqq 2^{n / 2} \exp \left[-\alpha^{2}\right] c_{1} / 2
\end{aligned}
$$

as long as $C \partial(x) \leqq \sqrt{t}$ because of Lemma 3.5.

The upper bound we announced is now an easy consequence of these estimates:

Proposition 3.7. Let us assume that the capacitary density of $\partial D$ is bounded below. Then, there exists a positive constants $C$ such that we have:

$$
Z(t) \leqq(2 \pi)^{-n / 2} \mathcal{Q}_{n}(D) t^{-n / 2}-(d)-m^{*}(\partial D) C t^{-d / 2}+o\left(t^{-d / 2}\right) .
$$

Proof. If $C$ and $c_{2}$ are the constants of Lemma 3.6, we have:

$$
\begin{aligned}
\int_{D} \mathbb{P}_{x}\left\{t>T_{D} \mid X_{t}=x\right\} d x & \geqq \int_{\{x \in D ; \partial(x) \leqq \sqrt{t / C}\}} \mathbb{P}_{x}\left\{t>T_{D} \mid X_{t}=x\right\} d x \\
& \geqq c_{2} \mathfrak{L}_{n}\{x \in D ; \partial(x) \leqq \sqrt{t} / C\},
\end{aligned}
$$

so that:

$$
\liminf _{t \rightarrow 0} t^{-(n-d) / 2} \int_{D} \mathbb{P}_{x}\left\{t>T_{D} \mid X_{t}=x\right\} d x \geqq c_{2} C^{(n-d) / 2}(d)-m_{*}(\partial D),
$$

and we conclude the proof like in Proposition 3.3.

We now show that the constants $c_{+}(n, d)$ and $c_{-}(n, d)$ are equal when the boundary $\partial D$ is $C^{1}$. But first we need the following estimate:

Lemma 3.8. If $\partial D$ is $C^{1}$, for each $\varepsilon>0$, there exists $d_{\varepsilon}>0$ such that:

$$
(1-\varepsilon) \exp \left[-2(1+\varepsilon)^{2} \partial(x)^{2} / t\right] \leqq \mathbb{P},\left\{T_{D}<t \mid X_{t}=x\right\} \leqq(1+\varepsilon) \exp \left[-2(1-\varepsilon)^{2} \partial(x)^{2} / t\right]
$$

whenever $\partial(x) \leqq d_{\varepsilon}$ and $t>0$.

Proof. We first prove the upper bound. Let $\varepsilon>0$ and $C>0$ be fixed. Without any loss of generality we can assume that $x$ is such that:

$$
\partial(x)^{2} / t>C_{\varepsilon}=[\log (1+\varepsilon)] /\left[2(1-\varepsilon)^{2}\right]
$$

since the upper bound is trivial otherwise.

Since $D$ is bounded and $\partial D$ is $C^{1}$, there exists $d_{0}=d_{0}(\varepsilon, C)>0$ such that for each $x$ in $D$ such that $\partial(x)<d_{0}$ one can find $y=y(x)$ in $\partial D$ such that $\|x-y\|=\partial(x)$ and such that the hypercube $R(x)$ containing $x$ and which is the intersection of the $2 n$ half-spaces $\Pi_{1}, \Pi_{2}, \ldots, \Pi_{2 n}$ limited by the planes $P_{1}, P_{2}, \ldots, P_{2 n}$ with $P_{1}$ and $P_{2}$ orthogonal to $x-y$ while $P_{2 j-1}$ and $P_{2 j}$ are parallel for $j=2, \ldots, n$ and orthogonal to $P_{2 k-1}$ and $P_{2 k}$ if $k \neq j$, and such that the distances $\partial_{j}(x)$ from $x$ to the planes $P_{j}$ 
satisfy $\partial_{1}(x)=(1-\varepsilon) \partial(x)$ and $\partial_{j}(x)=C \partial(x)$ for $j=2,3, \ldots, 2 n$. Now we have:

$$
\begin{aligned}
\mathbb{P}_{x}\left\{T_{D}<t \mid X_{t}=x\right\} & \leqq \mathbb{P}_{x}\left\{T_{R(x)}<t \mid X_{t}=x\right\} \\
& \leqq \sum_{1 \leqq i \leqq 2 n} \mathbb{P}_{x}\left\{T_{\Pi i}<t \mid X_{t}=x\right\} \\
& \leqq \exp \left[-2(1-\varepsilon)^{2} \partial(x)^{2} / t\right]+(2 n-1) \exp \left[-2 C^{2} \partial(x)^{2} / t\right] \\
& \leqq \exp \left[-2(1-\varepsilon)^{2} \partial(x)^{2} / t\right](1+(2 n-1) \\
& \cdot \exp \left[-2\left(C^{2}-2(1-\varepsilon)^{2}\right) \partial(x)^{2} / t\right]
\end{aligned}
$$

if one recalls (3.21), and:

$$
\leqq(1+\varepsilon) \exp \left[-2(1-\varepsilon)^{2} \partial(x)^{2} / t\right]
$$

if one chooses $C$ large enough. This is the expected upper bound. To prove the lower bound we note that, if we set $S(x)=\partial R^{\prime}(x)^{\prime} P_{1}$, where $R^{\prime}(x)$ is defined in the same way as $R(x)$ except for $\partial_{1}(x)$ which we take now as $\partial_{1}(x)=(1+\varepsilon) \partial(x)$, then the boundedness of $D$ and the fact that $\partial D$ is $C^{1}$ imply the existence of $d_{0}^{\prime}=d_{0}^{\prime}(\varepsilon, C)>0$ such that $S(x)$ is always contained in $D^{0}$. Consequently:

$$
\mathbb{P}_{x}\left\{T_{D}<t \mid X_{t}=x\right\} \geqq \mathbb{P}_{x}\left\{T_{S(x)}<t \mid X_{t}=x\right\}
$$

and the desired lower bound in an easy consequence of the following lemma and a simple scaling argument

Lemma 3.9. Let $x=(1,0, \ldots, 0)$ and $\Pi=\{0\} \times \mathbb{R}^{n-1}$. Then, for each $\varepsilon>0$ there exists an $\alpha>0$ such that the hypercube $J(\alpha)=\{0\} \times[-\alpha,+\alpha]^{n-1}$ in $\Pi$ satisfies:

$$
\mathbb{P}_{x}\left\{\tau_{J(\alpha)}<t \mid X_{t}=x\right\} \geqq(1-\varepsilon) \mathbb{P}_{x}\left\{T_{\Pi}<t \mid X_{t}=x\right\}=(1-\varepsilon) e^{-2 / t}
$$

for all $t>0$.

Proof. (3.22) rewrites:

$$
\mathbb{P}_{x}\left\{\tau_{J(\alpha)}<t \mid T_{\Pi}<t, X_{t}=x\right\} \geqq(1-\varepsilon),
$$

which is equivalent to:

$$
\mathbb{P}_{x}\left\{\tau_{J(\alpha)}<t \mid X_{t}=y\right\} \geqq(1-\varepsilon)
$$

with $y=(-1,0, \ldots, 0)$ because of the de André reflection principle. We first choose $a, T$, and $R$ such that:

$$
\mathbb{P}_{x}\{A\} \geqq 1-\varepsilon / 2,
$$

where we set $A=\left\{\tau_{J(a)}<t,\left\|X_{T}-y\right\| \leqq R\right\}$. Then, for all $t>T$ and $\alpha \geqq a$ we have:

$$
\begin{aligned}
& \mathbb{P}_{x}\left\{\tau_{J(\alpha)}<t \mid X_{t}=y\right\} \geqq \mathbb{P}_{x}\left\{\tau_{J(a)}<t,\left\|X_{T}-y\right\| \leqq R \mid X_{t}=y\right\} \\
& \quad=[t /(t-T)]^{n / 2} \mathbb{E}_{x}\left\{\mathbb{1}_{A} \exp \left[-\left|X_{T}-y\right|^{2} / 2(t-T)\right]\right\} \exp \left[|x-y|^{2} / 2 t\right] \\
& \quad \geqq \mathbb{P}_{x}\{A\} \exp \left[-R^{2} / 2(t-T)\right] \\
& \quad \geqq(1-\varepsilon)
\end{aligned}
$$


whenever $t \geqq T^{\prime}$ for some large enough constant $T^{\prime}$. Consequently, we need only prove (3.23) for $t<T^{\prime}$ and $\alpha$ large enough. But we also have:

$$
\mathbb{P}_{x}\left\{\tau_{J(\alpha)}<t \mid X_{t}=y\right\} \geqq \mathbb{P}_{x}\left\{T_{I(\alpha)}>t \mid X_{t}=y\right\},
$$

where we set $I(\alpha)=\mathbb{R} \times[-\alpha,+\alpha]^{n-1}$, and according to a calculation similar to a one we already made, we have:

$$
\begin{aligned}
& \geqq 1-2(n-1) \exp \left[-2 \alpha^{2} / t\right] \\
& \geqq 1-2(n-1) \exp \left[-2 \alpha^{2} / T^{\prime}\right] \\
& \geqq(1-\varepsilon),
\end{aligned}
$$

provided $\alpha$ is large enough. This concludes the proof.

In the very same way we proved Propositions 3.1 and 3.3 we obtain:

Proposition 3.10. If the boundary of $D$ is $C^{1}$ we have:

$$
Z(t)=(2 \pi)^{-n / 2} \mathfrak{Q}_{n}(D) t^{-n / 2}-(2 \pi)^{-(n-1) / 2} 2^{-3 / 2} \mathfrak{Q}_{n-1}(\partial D) t^{-(n-1) / 2}+o\left(t^{-(n-1) / 2}\right) .
$$

Acknowledgements. This work was done during the Spring quarter of 1984. The first name author would like to thank the Department of Mathematics of the University of California at Irvine for its warm hospitality and financial support. On the other hand, the second name author would like to thank R. Reilly and especially P. B. Gilkey for introducing him to the literature on the subject.

The original dedication was: "To Mark Kac, on the occasion of his $70^{\text {th }}$ birthday," "avec le plus protond respect et la plus grande admiration."

\section{References}

Berry, M.V.: Distribution of modes in fractals resonators. In: Structural stability in physics, pp. 51-53. Güttinger,W., Eikemeier, H. (eds.). Berlin, Heidelberg, New York: Springer 1979

Berry, M.V.: Some geometrical aspects of wave motion: wavefront, dislocations, diffraction catastrophes, diffractals. In: Geometry of the Laplace operator, Proc. Symp. Pure Math., Vol. 36, pp. 13-38. Providence RI: Am. Math. Soc. 1980

Berard, P.H.: Remarques sur la conjecture de Weyl. Composito Math. 48, 35-53 (1983)

Brownell, F.H.: Extended asymptotic eigenvalue distributions for bounded domain in $n$-space. J. Math. Mech. 6, 119-166 (1957)

Clark, C.: The asymptotic distributions of eigenvalues and eigenfunctions for elliptic boundary value problems. SIAM Rev. 9, 627-646 (1967)

Federer, H.: Geometric measure theory. Berlin, Heidelberg, New York: Springer 1969

Gromes, D.: Über die asymptotische Verteilung der Eigenwerte des Laplace Operators für Gebiete auf der Kugeloberfläche. Math. Z. 94, 110-121 (1966)

Ivrii, V.Ja.: Second term of the spectral asymptotic expansion of the Laplace-Beltrami operator on manifolds with boundary. Funct. Anal. Appl. 14, 98-106 (1980)

Kac, M.: Can you hear the shape of a drum? Am. Math. Mon. 73, 1-23 (1966)

Kuznetsov, N.V.: Asymptotic distribution of the eigenfrequencies of a plane membrane in the case when the variables can be separated. Differ. Equations 2, 715-723 (1966)

Louchard, G.: Mouvement brownien et valeurs propres du Laplacien. Ann. Inst. H.Poincaré Ser. B. 4, 331-342 (1968)

Mandelbrot, B.B.: Fractals: form, chance, and dimension. San Francisco: Freeman 1977

McKean, H.P., Singer, I.M.: Curvature and the eigenvalues of the Laplacian. J. Differ. Geom. 1, 43-69 (1967)

Melrose, R.: Weyl's conjecture for manifolds with concave boundary. In: Geometry of the Laplace operator. Proc. Symp. Pure Math., Vol. 36, pp. 254-274. Providence, RI: Am. Math. Soc. 1980 
Oksendale, B.K.: Null sets for measures orthogonal to $R(X)$. Am. J. Math. 44, 331-342 (1972) Port, S., Stone, C.: Brownian motion and classical potential theory. New York: Academic Press 1978

Seeley, R.: A sharp asymptotic remainder estimate for the eigenvalues of the Laplacian in a domain of $\mathbb{R}^{3}$. Adv. Math. 29, 244-269 (1978)

Simon, B.: Functional integration and quantum physics. New York: Academic Press 1979

Urakawa, H.: Bounded domains which are isospectral but not congruent. Ann. Sci. Ec. Norm. Super. 15, 441-456 (1982)

van den Berg, M.: Bounds on Green's functions of second order differential equations. J. Math. Phys. 22, 2452-2455 (1981)

Vitushkin, A.G.: Analytic capacity of sets and problems in approximation theory. Russ. Math. Surv. 18, 508-512 (1967)

Communicated by B. Simon

Received June 11, 1985; in revised form October 3, 1985 